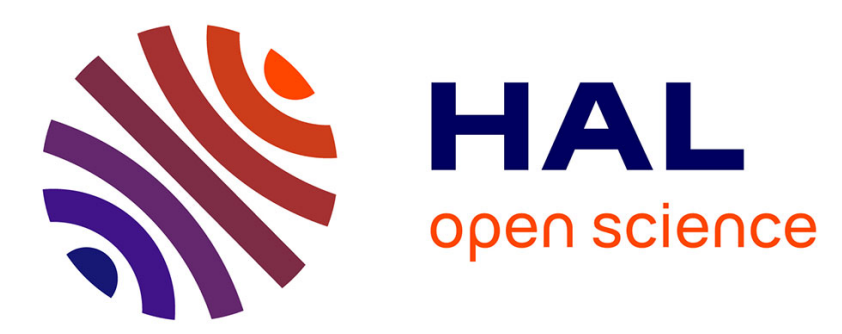

\title{
Influence of Biaxial Stress on Magnetic Behavior of Dual-Phase Steel - Experiments and Modeling
}

Olivier Hubert, Frédérick Sorel Mballa-Mballa, Song He, Sophie Depeyre

\section{To cite this version:}

Olivier Hubert, Frédérick Sorel Mballa-Mballa, Song He, Sophie Depeyre. Influence of Biaxial Stress on Magnetic Behavior of Dual-Phase Steel - Experiments and Modeling. IEEE Transactions on Magnetics, 2016, 52, pp.1 - 4. 10.1109/TMAG.2015.2511169 . hal-01532878

\section{HAL Id: hal-01532878 https://hal.science/hal-01532878}

Submitted on 3 Jun 2017

HAL is a multi-disciplinary open access archive for the deposit and dissemination of scientific research documents, whether they are published or not. The documents may come from teaching and research institutions in France or abroad, or from public or private research centers.
L'archive ouverte pluridisciplinaire HAL, est destinée au dépôt et à la diffusion de documents scientifiques de niveau recherche, publiés ou non, émanant des établissements d'enseignement et de recherche français ou étrangers, des laboratoires publics ou privés. 


\title{
Influence of biaxial stress on magnetic behavior of dual-phase steel - experiments and modeling
}

\author{
Olivier Hubert*, Frédérick Sorel Mballa-Mballa*, Song $\mathrm{He}^{\dagger}$ and Sophie Depeyre ${ }^{\dagger}$ \\ * LMT-Cachan (ENS Cachan, CNRS (UMR8535), Univ. Paris Saclay), 61 avenue du Président Wilson 94235 Cachan, France \\ $\dagger$ Technology Lab, Pôle Universitaire Léonard de Vinci, 92916 Paris La Défense Cedex, France \\ Email: hubert@1mt.ens-cachan.fr
}

\begin{abstract}
Online magnetic NDE of DP steels is made on bands submitted to high multiaxial stress. Extraction of microstructural information from the measurement requires separating the effect of stress and microstructure. Coupled magneto-mechanical micromagnetic modeling is relevant for that purpose. In this paper, magnetic behavior of DP steel under biaxial stress is carried out and compared to the modeling. It is shown that the discrepancies observed between experiments and model can be explained by the 2D assumption.
\end{abstract}

\section{INTRODUCTION}

Last few years a growing interest of car industry for the use of high performance steels as dual phase (DP) steels has been observed. The high mechanical performance of these steels (high strength, high ductility) is intimately linked to the presence of martensite hard phase embedded inside a ferritic phase matrix and strongly dependent on its volume fraction and distribution. The mechanical behavior is consequently highly sensitive to the thermo-mechanical history of the material and especially to small variations in the process (e.g. furnace temperature, scrolling speed). Magnetic online non-destructive monitoring techniques are consequently relevant to control the process [1]. However the non-destructive measurement is usually carried out on a band submitted to multiaxial (biaxial) stress state of high magnitude (tens of $\mathrm{MPa}$ ). The extraction of microstructural information from the measurement requires separating the effect of stress and of microstructure taking the possible couplings between stress and microstructure into account. We consequently must define a procedure to evaluate the effect of biaxial stress on material on the one hand, develop a modeling of this effect on the other hand, to finally observe the influence of each parameter and give the key for a future inverse identification. In this work, some results of the effect of biaxial stress on the magnetic behavior of a DP steel are first presented and discussed. A 2D coupled magneto-mechanical micro-magnetic modeling is next introduced. This model is able to take the microstructural features (distribution, shape of martensite and crystallographic texture) and multiaxial stress into account. This model is tested in biaxial mechanical configuration and compared to experimental results.

\section{MATERIAL AND EXPERIMENTAL PROCEDURE}

A $2.5 \mathrm{~mm}$ thick DP600 from Arcelormittal has been considered for the study. Figure 1 illustrates the microstructure of the material, exhibiting the martensite phase in white and ferrite phase in black. This microstructure is obtained by quenching of a low carbon steel (wt $\% \mathrm{C} \leq 0.3 \%$ typically) from austenoferritic domain of the phase diagram after several steps of hot and cold rolling.

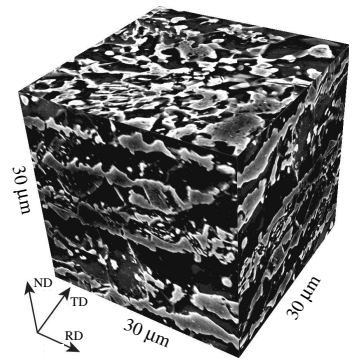

Fig. 1. Dual-phase steel microstructure - RD,TD,ND: rolling, tranverse and normal directions.

The basic idea of biaxial tests is to perform a measurement of magnetic behavior on a specimen loaded in tensioncompression along two perpendicular directions [2], [3], [4]. For that purpose, a cross-shaped specimen has been designed and cut from a $300 \times 300 \mathrm{~mm}^{2}$ DP600 sheet (general design reported in [2]). Compression tests can be carried out because the specimen is designed to prevent buckling. The local stress tensor $\hat{\sigma}=\left(\sigma_{1}, \sigma_{2}\right)$ in the centre of the specimen can be calculated from the forces applied along the two loading axes $\hat{F}=\left(F_{1}, F_{2}\right)$ according to an interacting symmetric matrix $\mathbf{A}$ $(\hat{\sigma}=\mathbf{A} . \hat{F})$. The terms of the matrix have been computed thanks to a previous finite element mechanical modeling of the specimen $\left(A_{11}=A_{22}=0.0078 \mathrm{MPa} / \mathrm{N}\right.$ and $A_{12}=A_{21}=-$ $0.0018 \mathrm{MPa} / \mathrm{N})$. The magnetic measurement area is a $15 \mathrm{~mm}$ diameter circle where both stress and magnetic field are fairly uniform [3] (heterogeneity level is estimated after finite element calculation less than $5 \%$ ).

The specimen is put horizontally between the jacks of the hydraulic machine ASTREE (figure 2a). Magnetic field and magnetic induction are measured thanks to a calibrated $\mathrm{H}$ coil (1000 turns coil - 15mm long, 8mm wide) and a needleB sensor (two parallel spikes to $15 \mathrm{~mm}$ from one to another, equipped with a spring that provides repeatable contact with the surface) respectively, put on the topside of the specimen 
(the spatial resolution is a circle of $15 \mathrm{~mm}$ diameter). Strain field is obtained thanks to Digital Image Correlation (DIC) on the bottom face of the specimen [5][6].

The experimental measurements reported in this work reduce to the measurements of initial susceptibility of anhysteretic curves $\chi_{i}\left(\sigma_{1}, \sigma_{2}\right)$. It allows a simple record of results (only one scalar value by mechanical state) and a fast comparison to modeling. Anhysteretic curves are constructed point after point by applying to the material an alternating magnetic field $(\mathrm{f}=2 \mathrm{~Hz})$ of decreasing amplitude superimposed on a constant bias magnetic field. The amplitude of the alternating field is slowly reduced to zero until only the bias field remains. The latter point defines the anhysteretic response corresponding to the applied bias field. The material is demagnetized after each measurement of anhysteretic point to eliminate the influence of loading history in the measurement.

The experimental procedure consist in several stress conditions $\left(\sigma_{1}, \sigma_{2}\right)$. The magnetic field is applied in the direction parallel to axis 1.24 biaxial loading configurations have been tested, for stress level varying from $-100 \mathrm{MPa}$ to $+100 \mathrm{MPa}$ (values defined after previous 1D experiments to meet the highest sensitivity). These stress states are plotted in the $\left(\sigma_{1}, \sigma_{2}\right)$ plane in figure $2 \mathrm{~b}$. Mechanical loading can be divided into parallel uniaxial tests $\left(\sigma_{1} \neq 0, \sigma_{2}=0\right)$, orthogonal uniaxial tests $\left(\sigma_{1}=0, \sigma_{2} \neq 0\right)$, equibiaxial tests $\left(\sigma_{1}=\sigma_{2}\right)$, and shear tests $\left(\sigma_{1}=-\sigma_{2}\right)$ in order to map the stress plane.
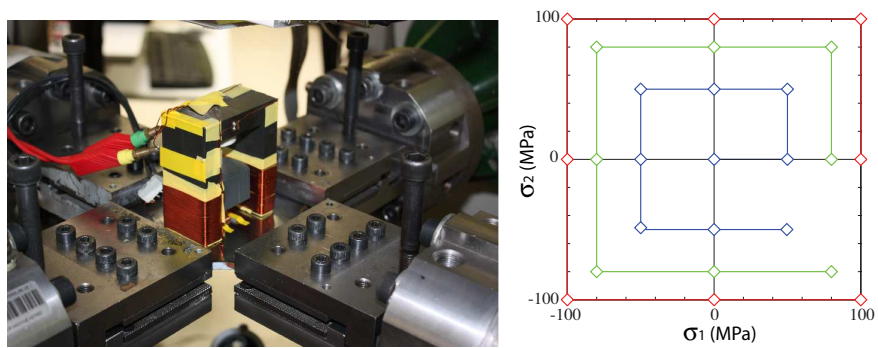

Fig. 2. (a) benchmark for magnetic measurement under biaxial stress; (b) stress states used for experimental measurements plotted in the $\left(\sigma_{1}, \sigma_{2}\right)$ plane.

\section{MAGnetomeCHANICAL MiCROMAGNETIC MODELING}

Micromagnetism is a theoretical approach to describe the process of magnetization at a scale large enough to replace the atomic magnetic moments by continuous functions, and small enough to account for the transition zones between magnetic domains [7][8]. Approximation is obtained thanks to the minimization of an energy functional. The contribution of Brown was to define an expression of the free energy $\mathscr{E}_{\text {tot }}$ (1) as function of the local magnetic moment $\vec{m}$. $\mathscr{E}_{\text {tot }}$ is the sum over a volume $\Omega$ of internal and external contributions: the exchange energy $\mathscr{E}_{e x}$, the magnetocrystalline energy $\mathscr{E}_{a}$, the Zeeman energy $\mathscr{E}_{h}$ and the dipole interaction $\mathscr{E}_{d}$ (full expressions are not detailed herein - please report to [9] for more details).

$$
\mathscr{E}_{\text {tot }}(\vec{m})=\mathscr{E}_{h}+\mathscr{E}_{e x}+\mathscr{E}_{a}+\mathscr{E}_{d}
$$

Introduction of the mechanical energy (and associated magnetoelastic effects) into this expression has been made in a recent work [9]. It allows the modeling of the magnetostrictive behavior and the influence of stress on the magnetic behavior (full magnetoelastic coupling). The main characteristics of this modeling are recalled hereafter.

The change of magnetization of a magnetic medium induces a deformation $\varepsilon^{\mu}$ called magnetostriction. The magnetostriction strain tensor for cubic symmetry is isochore and given by:

$$
\varepsilon^{\mu}=\frac{3}{2}\left(\begin{array}{ccc}
\lambda_{100}\left(\alpha_{1}^{2}-\frac{1}{3}\right) & \lambda_{111} \alpha_{1} \gamma_{2} & \lambda_{11 \alpha 1} \alpha_{1} \alpha_{3} \\
\lambda_{111} \alpha_{1} \alpha_{2} & \lambda_{100}\left(\alpha_{2}^{2}-\frac{1}{3}\right) & \lambda_{111} \alpha_{2} \alpha_{3} \\
\lambda_{111} \alpha_{1} \alpha_{3} & \lambda_{111} \alpha_{2} \alpha_{3} & \lambda_{100}\left(\alpha_{3}^{2}-\frac{1}{3}\right)
\end{array}\right)
$$

where $\lambda_{100}$ and $\lambda_{111}$ are two magnetostrictive constants. Since this deformation is mechanically incompatible (isochore), an elastic deformation $\varepsilon^{\ell}$ of the magnetic medium is always associated in case of embedded inclusion. It results to a stress field even in absence of any external mechanical loading. When the magnetostriction $\varepsilon^{\mu}$ is known for a medium, the total deformation $\varepsilon$ is obtained by simple addition $\left(\varepsilon=\varepsilon^{\mu}+\varepsilon^{e}\right)$ since small pertubations assumption can be applied. The total deformation $\varepsilon$ derives from a displacement field $\vec{u}(3)$ and stress field $\sigma$ associated to elastic deformation obeys to the local linear momentum balance equation (4) with $\mathbb{C}$ the stiffness tensor of the medium.

$$
\begin{gathered}
\varepsilon=\frac{1}{2}\left(\overline{\bar{\nabla}} \vec{u}+{ }^{t} \overline{\bar{\nabla}} \vec{u}\right) \quad \text { in } \Omega \\
\vec{\nabla} \cdot \sigma=\overrightarrow{0} \quad \text { in } \Omega \\
\sigma=\mathbb{C}: \varepsilon^{e} \text { in } \Omega
\end{gathered}
$$

The boundary conditions of the problem are given by:

$$
\begin{gathered}
\sigma \cdot \vec{n}=\vec{T}_{d} \quad \text { on } \quad \partial \Omega_{t} \\
\vec{u}=\vec{u}_{d} \quad \text { on } \quad \partial \Omega_{u}
\end{gathered}
$$

$\vec{T}_{d}$ and $\vec{u}_{d}$ are the surface forces and displacements applied at the boundaries (surfaces) $\partial \Omega_{t}$ and $\partial \Omega_{u}$ respectively. The additivity of deformations allows to reformulate the mechanical balance to introduce an internal stress of magnetostrictive origin:

$$
\vec{\nabla} \cdot \sigma^{\star}-\vec{f}^{\mu}=\overrightarrow{0}
$$

$\sigma^{\star}$ is the total stress and $\vec{f}^{\mu}=\vec{\nabla}\left(\mathbb{C}: \varepsilon^{\mu}\right)$ the force density of magnetostrictive origin. The mechanical problem can also be reduced to an optimization problem where the displacement field minimizes the elastic energy $\mathscr{E}_{\sigma}$ given by the application of a variational formulation of the problem (6)

$$
\vec{u}=\operatorname{Min}_{\left(\vec{v} \in \mathscr{H}_{\Omega}^{1}\right)} \mathscr{E}_{\sigma}(\vec{v}) \quad \text { with } \vec{v}=\vec{u}_{d} \quad \text { on } \quad \partial \Omega_{d}
$$

with:

$$
\mathscr{E}_{\sigma}(\vec{v})=\frac{1}{2} \varepsilon(\vec{v}): \mathbb{C}: \varepsilon(\vec{v})-\varepsilon(\vec{v}): \mathbb{C}: \varepsilon^{\mu}
$$


Considering a magneto-mechanical coupled approach, contributions related to magnetic equilibrium, but also contributions related to the mechanical equilibrium and various interactions between these two phenomena are involved in the energy equilibrium. The free energy that suitably describes a deformable magnetic medium takes the following form:

$$
\mathscr{E}_{t o t}(\vec{m}, \vec{u})=\mathscr{E}_{h}+\mathscr{E}_{e x}+\mathscr{E}_{a}+\mathscr{E}_{d}+\mathscr{E}_{\sigma}
$$

The stability condition of the energy is obtained if and only if the magnetization and displacement fields minimize the total free energy simultaneously.

A condition of minimization is the cancellation of all the partial derivatives independently. We solve the system:

$$
\frac{\partial \mathscr{E}_{t o t}}{\partial \vec{m}}=\overrightarrow{0} \quad \frac{\partial \mathscr{E}_{t o t}}{\partial \vec{u}}=\overrightarrow{0} \quad \forall \vec{x} \in \Omega
$$

Numerical methods used for the resolution and associated algorithms are detailed in [9].

\section{EXPERIMENTAL RESULTS AND COMPARISON TO MODELING}

Figure 3 illustrates the evolution of the experimental initial susceptibility in the stress plane. The susceptibility under stress is normalized by the value of the susceptibility at the same magnetic field level at zero stress $\left(\chi_{0}=3200\right)$. The main observations are: uniaxial tension along the magnetic field direction improves the susceptibility and a compression in the same direction deteriorates it. When a uniaxial stress is applied in the direction perpendicular to the magnetic field, the effect is opposite and attenuated. The tension-compression asymmetry is very perceptible: bi-tension does not modify significantly the susceptibility, while bi-compression decreases it. The lowest and highest values of $\chi_{i}$ are obtained for a shear situation: lowest for $\sigma_{1}<0$ and highest for $\sigma_{1}>0$. These results meet some previous results reported for ironcobalt [4] and more recently for iron-silicon [5]. Indeed all these materials exhibit positive magnetostriction. Considering an average magnetostriction $\lambda>0$, the magnetoelastic energy for magnetic field applied along direction $\overrightarrow{1}$ can be roughly expressed as: $W_{m e}=-\sigma_{1} \lambda+\sigma_{2} \lambda / 2$ [3], leading to equal behavior lines of slope 2 in the $\left(\sigma_{1}, \sigma_{2}\right)$ plane and higher susceptibility for $\sigma_{1}>0$ (lower energy). Non-linearity of behaviors leads to the curvature of equal lines.

Micromagnetic code "sivimm $2 d$ " [10] is used as the basis for the implementation of the magneto-mechanical formulation. For simplicity purpose, we only considered the evolution of initial susceptibility of a single crystal (axes: $[100]=\overrightarrow{1}$ and $[010]=\overrightarrow{2}$ ). The region of interest is a square of $30 \times 30 \mu \mathrm{m}^{2}$ in the vacuum. This region is meshed by 3996 triangular elements. Magnetic and mechanical constants are reported on table I $\left(M_{s}\right.$ is the saturation magnetization; $A, K_{i}$ and $C_{i j}$ are the exchange,magnetocrystalline and stiffness constants). The domain distribution after minimization of the full energy functional without any external loading is given in figure 4a.

To reproduce the experimental conditions, we apply suitable boundaries conditions (BC) on the sides of the square. These

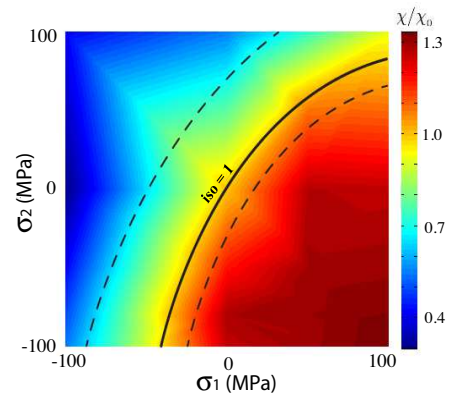

Fig. 3. Evolution of normalized anhysteretic initial susceptibility $\chi_{i} / \chi_{0}$ (interpolation) in the $\left(\sigma_{1}, \sigma_{2}\right)$ plane - with $\chi_{0}=3200$

\begin{tabular}{|c|c|c|}
\hline$M_{s}=1.71 \times 10^{6} \mathrm{~A} . \mathrm{m}^{-1}$ & $A=1.8 \times 10^{-11}{\mathrm{~J} . \mathrm{m}^{-1}}^{-1}$ & $K_{1}=48 \times 10^{3}{\mathrm{~J} . \mathrm{m}^{-3}}^{-6}$ \\
$K_{2}=0 \mathrm{~J} . \mathrm{m}^{-3}$ & $\lambda_{100}=21 \times 10^{-6}$ & $\lambda_{111}=-21 \times 10^{-6}$ \\
$C_{11}=228 \mathrm{GPa}$ & $C_{12}=132 \mathrm{GPa}$ & $C_{44}=116.5 \mathrm{GPa}$ \\
\hline
\end{tabular}

TABLE I

PHYSICAL CONSTANTS USED FOR SIMULATIONS

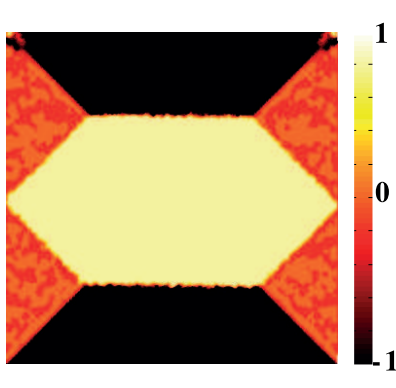

(a)

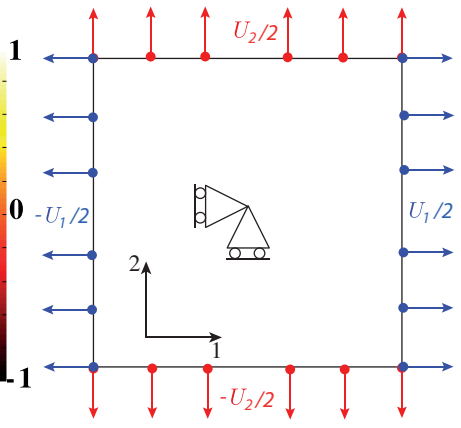

(b)

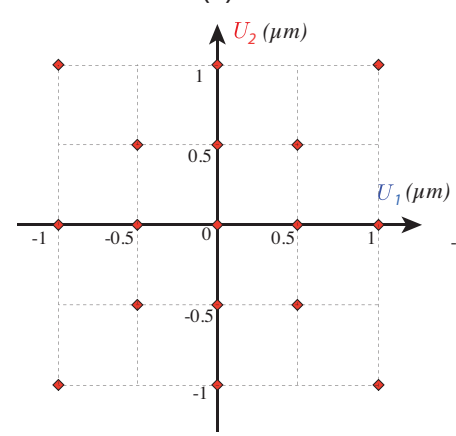

(c)

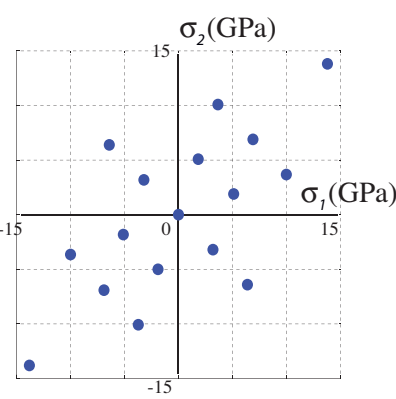

(d)
Fig. 4. (a) Initial domain definition: color code corresponding to $m_{1}=(\vec{m} . \overrightarrow{1})$; (b) boundary conditions used for the modeling; (c) loadings $\left(u_{1}, u_{2}\right)$ considered for the modeling and (d) associated average stress.

conditions consist in different displacement magnitude $u_{1}$, $u_{2}$ applied along directions 1 and 2 (figures $4 \mathrm{~b}$ and $4 \mathrm{c}$ ). Stress $\sigma$ is then computed by averaging over the surface (figure 4d). Finally, a numerical anhysteretic procedure is applied at each mechanical point (20 demagnetization points) to calculate the corresponding numerical anhysteretic point and numerical initial susceptibility. 2D modeling leads to high demagnetization field and low mobility of magnetic moments: a $0.15 \times M_{s}$ bias field is consequently required to 
reach a $0.3 \times M_{s}$ magnetization level and calculate associated susceptibility. This bias field level is more than 100 times the level required during experiments. For similar reasons, the stress levels required to generate a significant effect on modeled magnetic behavior is 100 times higher than the stress level required during experiments [11]. Some large displacements must consequently be applied (magnitude of 1 $\mu \mathrm{m}$ to reach a stress level of about 10GPa). Figure 5 illustrates the evolution of the micromagnetic initial susceptibility in the stress plane (relatively to the zero stress value $-\chi_{0}=2$ before correction of demagnetizing field). This simulation is in accordance with the experimental results concerning the effect of shear stress, parallel and orthogonal uniaxial stress. The equibiaxial loading leads on the contrary to different results. The iso-susceptibility curves $\left(\chi_{i} / \chi_{0}=\right.$ cte $)$ seem corresponding to straight lines whereas the iso-susceptibility curves observed during experiments appear more curved.

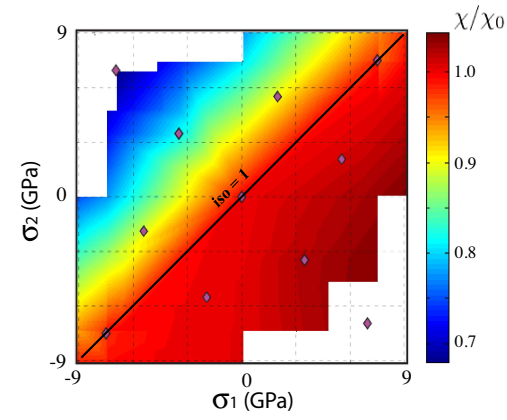

Fig. 5. Evolution of relative initial susceptibility $\chi_{i} / \chi_{0}$ (interpolation) in the $\left(\sigma_{1}, \sigma_{2}\right)$ plane obtained from the micromagnetic model - with $\chi_{0}=2$.

An equivalent stress model that has been proposed recently [4] confirms that the expected iso-susceptibility curves should exhibit a decreasing slope with increasing $\sigma_{2}$. Indeed, in the case of biaxial stress state $\left(\sigma_{1}, \sigma_{2}\right)$ and magnetic field applied along $\overrightarrow{1}$, the equivalent stress is given by:

$$
\sigma_{e q}=\frac{2}{3 k} \ln \left(\frac{2 \exp \left(\frac{3}{2} k \sigma_{1}\right)}{1+\exp \left(\frac{3}{2} k \sigma_{2}\right)}\right)
$$

with $k=\frac{6 \chi_{0} \lambda_{100}}{5 \mu_{0} M_{s}^{2}}$ ( $\mu_{0}$ : vacuum permeability). Results are plotted in figure 6. Evolutions of equivalent stress (form of isovalue curves) in the stress plane are in accordance with the experimental results.

It must be noticed that equation (11) has been derived using a modeling of ferromagnetic material considered as an assembly of six domains as illustrated in figure 7. But the micromagnetic simulations were performed using a $2 \mathrm{D}$ assumption for the distribution of magnetic moments. If we consider now an assembly of four domains, a new formulation of equivalent stress can be derived. After few calculation (using the same approach than in [4]), in the case of biaxial stress state $\left(\sigma_{1}, \sigma_{2}\right)$ and magnetic field applied along $\overrightarrow{1}$, the $2 \mathrm{D}$ equivalent stress is given by:

$$
\sigma_{e q}=\sigma_{1}-\sigma_{2}
$$

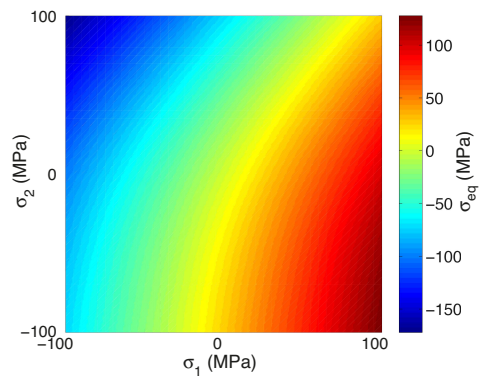

Fig. 6. Equivalent stress $\sigma_{e q}$ for $\left\{\sigma_{1}, \sigma_{2}\right\} \in[-100 \mathrm{MPa}, 100 \mathrm{MPa}]$ $\mathrm{k}=1.3716 \times 10^{-8} \mathrm{~Pa}^{-1}$.
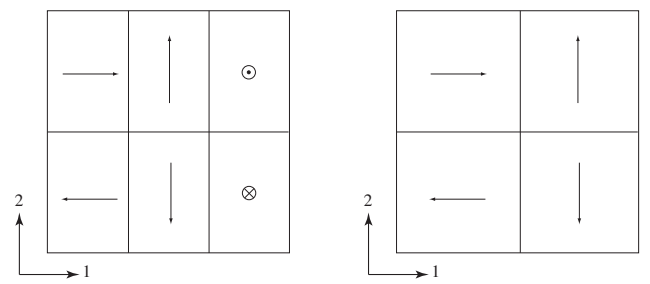

Fig. 7. Elementary representation of ferromagnetic material used for the derivation of equivalent stress: (a) six domains (3D configuration) [4]; (b) four domains (2D configuration).

This equivalent stress meets the equivalent stress proposed in the early work of Schneider [12]. Iso-values obtained are straight lines of slope 1, in accordance with the results from the micromagnetic modeling. The 2D distribution of the magnetic moments in the latter modeling seems clearly at the origin of shape of iso-susceptibility curves. This result question about the ability of the $2 \mathrm{D}$ micromagnetic to restitute properly the magneto-mechanical coupling.

\section{CONClusion}

The influence of biaxial stress on the magnetic anhysteretic susceptibility of dual-phase steel has been addressed from an experimental and a modeling point of view. It has been demonstrated that 3D magneto-mechanical approaches (analytic or micromagnetic) are required to simulate accurately the influence of multiaxial stress on the magnetic behavior.

\section{REFERENCES}

[1] G.Dobmann. Physical basics and industrial applications of $3 \mathrm{MA} \mathrm{mi}$ cromagnetic multiparameter microstructure and stress analysis. 12th Int. Workshop on Electromagnetic NDE (ENDE2007), Cardiff, UK, 2007.

[2] O.Hubert et al., Przedglad Elektrotecniczny R81, 5 (2005) 19-23.

[3] O.Hubert, Przedglad Elektrotecniczny R83, 4 (2007) 70-77.

[4] O.Hubert and L.Daniel, Journal of Magnetism and Magnetic Materials, 323, 20, (2011) 1766-1781.

[5] M.Rekik et al., International Journal of Applied Electromagnetics and Mechanics, 44, (3-4) (2014) 301-315.

[6] F.Hild et al., Applied Optics, 41(32) (2002) 6815-6828

[7] W.F.Brown. Micromagnetics, John Wiley \& Sons, New York, 1963.

[8] A.Aharoni Introduction to the Theory of Ferromagnetism. Oxford University Press, New York, 1996.

[9] F.Mballa et al., IEEE Transactions on Magnetics, 50, 4 (2014) 1-4.

[10] M.Bernadou et al., J. of intelligent material systems and structures, 16, (2005) 1015-1023.

[11] F. Mballa, PhD thesis, ENS-Cachan, France, 2013.

[12] C.S.Schneider and J.M.Richardson, Journal Applied Physics ,53 (1982) 8136-8138. 